\title{
On the Electric Dipole Moments of Asymmetric Tops: Measurement by High-Resolution Electronic Spectroscopy in the Gas Phase
}

\author{
Diane M. Miller, ${ }^{\dagger}$ Philip J. Morgan, and David W. Pratt* \\ Department of Chemistry, University of Pittsburgh, Pittsburgh Pennsylvania 15260
}

Received: February 25, 2009; Revised Manuscript Received: May 4, 2009

\begin{abstract}
Reported here are Stark-effect measurements of the permanent electric dipole moments of two structural isomers of aminobenzonitrile, $2 \mathrm{ABN}$ and $3 \mathrm{ABN}$, using high-resolution laser molecular beam techniques. When combined with previous results on $4 \mathrm{ABN}$, the data show that each structural isomer has a unique dipole moment, in both its ground $\left(\mathrm{S}_{0}\right)$ and electronically excited $\left(\mathrm{S}_{1}\right)$ states, thereby providing a means of distinguishing them. Possible applications of the method to other, biologically relevant molecules are discussed.
\end{abstract}

\section{Introduction}

The "molecular design of life" is significantly influenced by the properties of isolated molecules. Thus, the three-dimensional structures and functions of proteins depend upon the size, shape, and charge distributions of the side chains in the 20 different amino acid building blocks that are used to construct them. Protein-protein interactions, leading to aggregation and disease, are also believed by some to be influenced by the dipole moments of their component parts. ${ }^{2}$ It is thus a bit surprising that the dipole moments of the individual amino acids, and their dependence on the conformation of the isolated molecule, remain largely unexplored by experimental techniques.

Significant progress toward achieving this goal has been made, beginning with the work of Brown and co-workers on glycine, histamine, alanine, and related molecules a number of years ago. ${ }^{3}$ More recently, several neutral aliphatic amino acids in the gas phase have been studied using laser ablation, molecular beam Fourier transform microwave spectroscopy, ${ }^{4}$ and their preferred structures determined by measurements of their rotational and ${ }^{14} \mathrm{~N}$ quadrupole coupling constants. Mons and co-workers, ${ }^{5}$ and others, have probed the structures of small peptides using IR/UV double-resonance techniques. But the dipole moments of many of these species have yet to be reported. Compagnon et al. ${ }^{6}$ have coupled a matrix-assisted laser desorption source to an electric beam deflection setup to measure the permanent electric dipole of tryptophan isolated in a molecular beam. More recently, this method has been applied to a variety of species, including glycine-based ${ }^{7}$ and alaninebased peptides. ${ }^{8}$ In the latter work, the authors concluded that alanine-based peptides formed $\beta$-sheets in the gas phase, rather than the $\alpha$-helices normally observed in aqueous solution, because the observed dipole moments were much smaller than expected.

One possible explanation for this anomaly, offered first by Abd El Rahim et al., ${ }^{9}$ is that the gas-phase alanine-based peptides are actually helical in shape and that deviations from their expected deflection behaviors were a consequence of the asymmetry of the molecule. This idea was tested, and it was indeed found that, in a family of aminobenzonitrile ( $\mathrm{ABN}$ ) molecules, there were increasing deviations from the calculated

\footnotetext{
* Corresponding author. E-mail: pratt@pitt.edu.

Present address: Department of Chemistry, Marquette University, Milwaukee WI 53201.
}

Stark-effect behavior with increasing asymmetry in the series $4 \mathrm{ABN}, 3 \mathrm{ABN}$, and $2 \mathrm{ABN}$, shown below.

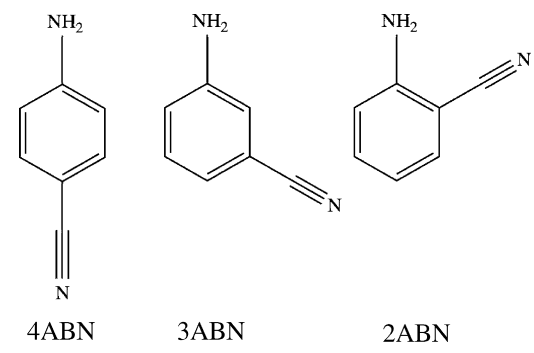

Described herein are the results of Stark-effect experiments by high-resolution laser spectroscopy on two of these molecules, $2 \mathrm{ABN}$ and $3 \mathrm{ABN}$. (Similar results for $4 \mathrm{ABN}$ have previously been reported. ${ }^{10}$ ) The objectives of this work were (a) to show that accurate values of the permanent electric dipole moments of these species could, in fact, be measured, (b) to compare the measured values with ab initio values upon which the calculations of Abd El Rahim, et al. ${ }^{9}$ were based, and therefore, (c) to validate (or invalidate) their explanation of the observed deflection behavior. We find that this explanation is correct, a fact that was anticipated by others. ${ }^{11}$ But we also show, as a consequence, that the method described here (as well as related microwave methods) can still be relied upon for future measurements of the dipole moments of the "building blocks of life". 1

\section{Experimental Section}

2ABN (98\% purity) and 3ABN (99\% purity) were purchased from Sigma-Aldrich and used as received. Vibrationally resolved fluorescence excitation spectra (FES) were obtained by heating the sample to $\sim 40{ }^{\circ} \mathrm{C}$, seeding the resulting vapor into $2-2.5$ kTorr of dry helium gas ( $>90 \%$ purity), and expanding the mixture through a $1 \mathrm{~mm}$ diameter orifice pulsed valve (General Valve series 9 , operating at $10 \mathrm{~Hz})$ into a vacuum chamber $\left(10^{-5}\right.$ Torr). The cooled sample was then crossed $2 \mathrm{~cm}$ downstream of the nozzle with the output of a Quanta-Ray $\mathrm{Nd}^{3+}$ :YAG (model DCR-1A) pumped dye laser (model PDL-1). DCM dye was used to obtain the correct visible laser frequency; this was then doubled externally with a potassium dihydrogen phosphate (KDP) crystal. Any remaining visible light was filtered, leaving ultraviolet (UV) light with a spectral resolution of $\sim 1 \mathrm{~cm}^{-1}$. 
Fluorescence was collected with a photomultiplier tube (EMI 98139B) positioned at right angles to both the laser and nozzle beams. The data were processed by a boxcar integrator (Stanford Research Systems) and recorded using Quick Data Acquisition software (version 1.0.5).

The molecular beam laser spectrometer used to obtain rotationally resolved spectra has been described elsewhere. ${ }^{12}$ Briefly, the samples were each heated to $\sim 120{ }^{\circ} \mathrm{C}$, seeded in dry argon gas ( $>99 \%$ purity), and expanded into a vacuum through a $240 \mu \mathrm{m}$ quartz nozzle. The molecular beam was formed by skimming the expansion $2 \mathrm{~cm}$ downstream of the nozzle with a $1 \mathrm{~mm}$ diameter skimmer and then crossed $15 \mathrm{~cm}$ downstream with the frequency-doubled output of a modified continuous ring dye laser. DCM dye was used for $2 \mathrm{ABN}$, and DCM Special dye was used for $3 \mathrm{ABN}$. The visible output of the dye laser was externally frequency-doubled by a SpectraPhysics Wavetrain using a $630 \mathrm{~nm}$ LBO crystal; ${ }^{13} \sim 400$ and $\sim 200 \mu \mathrm{W}$ of UV light was produced at the $2 \mathrm{ABN}$ and $3 \mathrm{ABN}$ origin frequencies, respectively. Fluorescence was collected using spatially selective optics and detected by a photomultiplier tube (EMI 9813 QB) and photon counting system. All information was processed using the jba95 data acquisition system. ${ }^{14}$ Absolute transition frequencies in the excitation spectrum were determined by comparison to the $\mathrm{I}_{2}$ absorption spectrum (accuracy $\sim 30 \mathrm{MHz}$ ), which was recorded simultaneously with the high-resolution spectrum. Frequency markers also were collected; these were generated by passing a small portion of the fundamental through a stabilized etalon with a free spectral range of $299.7520 \pm 0.0005 \mathrm{MHz}$. Fitting of the zero-field spectra was performed using the jb95 rotational least-squares fitting program. ${ }^{15}$ The experimental setup of the Stark cell and the procedure for analyses of Stark spectra have been described in detail elsewhere, ${ }^{16}$ applied field strengths ranged from $\sim 95$ to $1900 \mathrm{~V} / \mathrm{cm}$.

\section{Results}

Vibrationally resolved FES of 2ABN and 3ABN are shown in Figure 1. Both spectra are consistent with previously published data on the two molecules. ${ }^{17,18}$ In comparison to the FES of 4-aminobenzonitrile $(4 \mathrm{ABN}),{ }^{18}$ the spectra of $2 \mathrm{ABN}$ and $3 \mathrm{ABN}$ show more vibrational activity. $4 \mathrm{ABN}$ contains a symmetry plane and is a near symmetric top molecule $(\kappa=$ $-0.94) ; 2 \mathrm{ABN}$ and $3 \mathrm{ABN}$, to varying degrees, are less symmetric, allowing more transitions to be observed.

The rotationally resolved FES of the origin band of $2 \mathrm{ABN}$ at zero field is shown in Figure 2a. The band shows 44/55 a/b hybrid character ${ }^{19}$ with the transition moment lying between the $a$ and $b$ axes. Rotational constants obtained by ab initio calculations,$^{20}$ shown in Table 1, were used to create an initial simulation. Transitions were assigned and the rotational constants were adjusted until the simulation was in good agreement with the experimental spectrum. The parameters obtained from this fit are given in Table 1. A portion of the simulation is shown in the lower part of Figure 2a, demonstrating its good agreement with experiment. Each line of the simulation represents an individual $\left|J^{\prime} K_{a}{ }^{\prime} K_{c}\right\rangle \leftarrow\left|J^{\prime \prime} K_{a}{ }^{\prime \prime} K_{c}{ }^{\prime \prime}\right\rangle$ transition; 101 transitions were assigned. Width has been added to reproduce the overall shape of the experimental spectrum. The best fit had an OMC (observed minus calculated standard deviation) of $1.9 \mathrm{MHz}$. The measured rotational constants are in good agreement with theoretical ones, with differences of less than $1 \%$ for the ground state and less than $2 \%$ for the excited state.

Figure $2 \mathrm{~b}$ shows the corresponding rotationally resolved FES of the origin band of $3 \mathrm{ABN}$ at zero field. An initial simulation
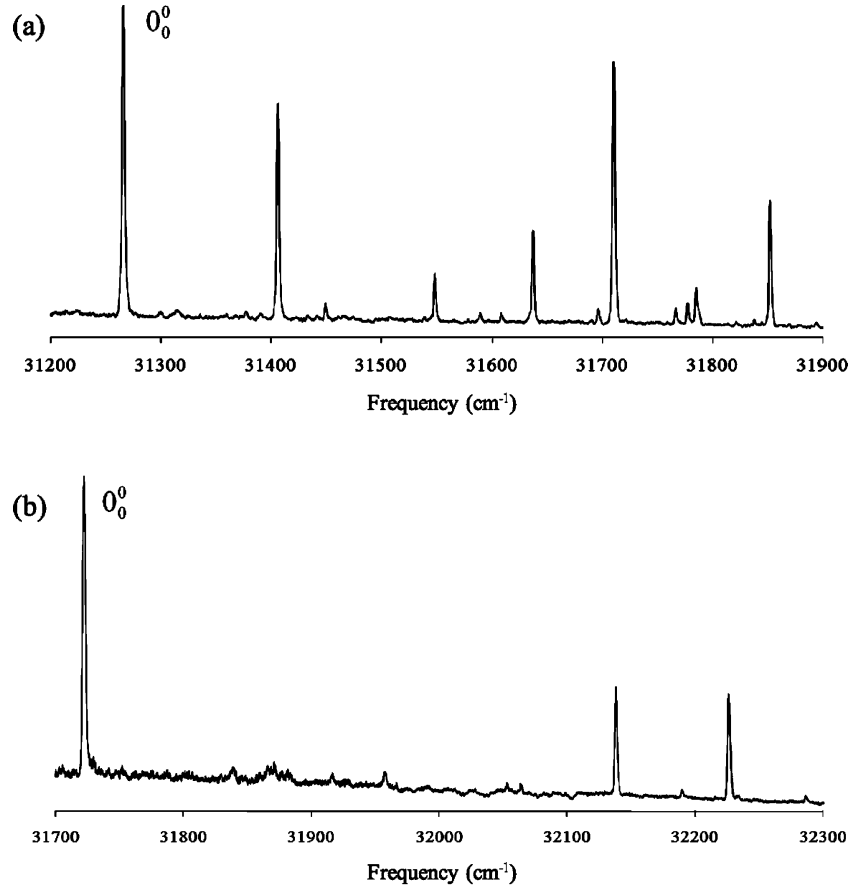

Figure 1. Vibrationally resolved fluorescence excitation spectra of $2 \mathrm{ABN}$ (a) and $3 \mathrm{ABN}(\mathrm{b})$.

was created using the ab initio values given in Table 1, and transitions were assigned and the rotational constants were adjusted until the simulation was in good agreement with the experimental spectrum. A portion of the best-fit simulation, with and without added line width, is shown in the lower portion of Figure 2b. Over 100 transitions were assigned; the best fit had an OMC of 4.6 MHz. The measured rotational constants are shown in Table 1, and they are again in good agreement with calculations, with differences of less than $1 \%$ for the ground state and less than $2 \%$ for the excited state. Fits of the spectral

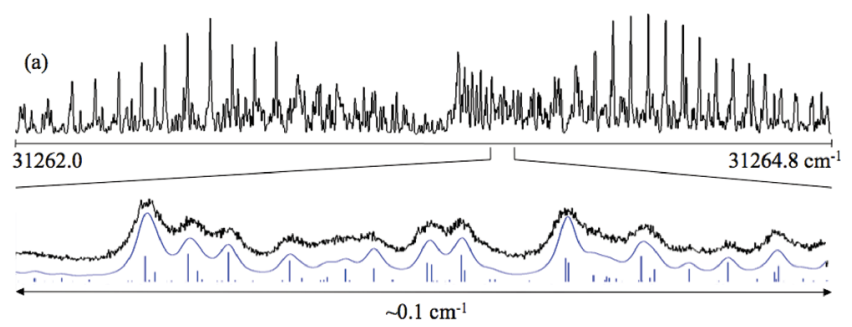

(b)

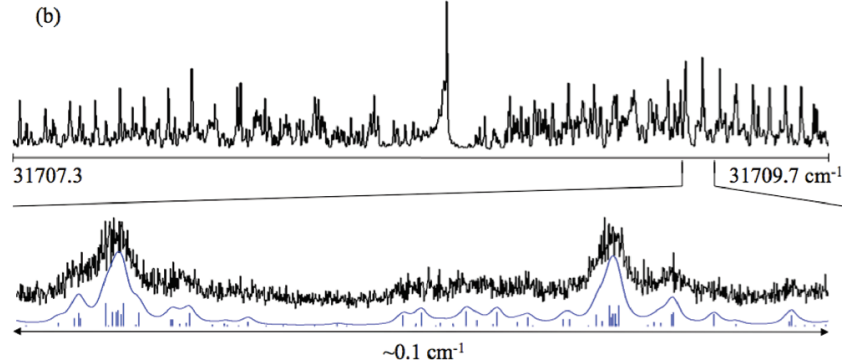

Figure 2. (a) Field-free origin band spectrum of $2 \mathrm{ABN}$, occurring at $31263.5 \mathrm{~cm}^{-1}$. The bottom panel shows a comparison of a small portion of the experimental spectrum (black trace) with two simulated spectra (blue trace), with and without a convoluted line shape function. (b) Field-free origin band spectrum of $3 \mathrm{ABN}$, occurring at $31708.6 \mathrm{~cm}^{-1}$. The bottom panel shows a comparison of a small portion of the experimental spectrum (black trace) with two simulated spectra (blue trace), with and without a convoluted line shape function. 
TABLE 1: Experimental Inertial Parameters of 2ABN and $\mathbf{3 A B N}^{a}$

\begin{tabular}{|c|c|c|c|c|c|c|}
\hline & \multicolumn{3}{|c|}{$2 \mathrm{ABN}$} & \multicolumn{3}{|c|}{$3 \mathrm{ABN}$} \\
\hline & experiment & theory & $\begin{array}{c}\text { error } \\
(\%)\end{array}$ & experiment & theory & $\begin{array}{c}\text { error } \\
(\%)\end{array}$ \\
\hline \multicolumn{7}{|c|}{$\mathrm{S}_{0}$} \\
\hline$A(\mathrm{MHz})$ & 3009.0 (1) & 2992.5 & 0.5 & $3372.7(1)$ & 3366.3 & 0.2 \\
\hline$B(\mathrm{MHz})$ & 1509.0 & 1499.1 & 0.6 & 1209.9 & 1201.1 & 0.7 \\
\hline$C(\mathrm{MHz})$ & $1005.2(1)$ & 999.8 & 0.5 & 890.8 (1) & 886.0 & 0.5 \\
\hline$\Delta I\left(\operatorname{amu} \AA^{2}\right)$ & -0.10 & -0.52 & & -0.22 & -0.50 & \\
\hline \multicolumn{7}{|c|}{3} \\
\hline$A(\mathrm{MHz})$ & 3093.8 (2) & 3085.6 & 0.3 & $3373.6(1)$ & 3435.1 & 1.8 \\
\hline$B(\mathrm{MHz})$ & $1464.4(1)$ & 1488.1 & 1.6 & $1189.4(1)$ & 1201.0 & 1.0 \\
\hline$C(\mathrm{MHz})$ & 994.1 (1) & 1003.9 & 1.0 & 879.5 (1) & 890.0 & 1.2 \\
\hline$\Delta I\left(\operatorname{amu} \AA^{2}\right)$ & -0.08 & 0.02 & & -0.08 & -0.10 & \\
\hline$a / b / c$ & $45 / 55 / 0$ & & & $25 / 75 / 0$ & & \\
\hline origin $\left(\mathrm{cm}^{-1}\right)$ & 31263.5 & & & 31708.6 & & \\
\hline
\end{tabular}

${ }^{a}$ Theoretical values calculated using the MP2/6-31G** and CIS/ 6-31G** methods also have been included for comparison.

line intensities are consistent with a band having 25/75 a/b hybrid character. This is substantially different from the band character seen for $2 \mathrm{ABN}$.

Figure 3 shows the spectrum of $2 \mathrm{ABN}$ in the presence of an electric field of $950 \mathrm{~V} / \mathrm{cm}$. To simulate the behavior of the spectrum in the presence of an applied electric field, a special program ${ }^{16}$ was used, in conjunction with jb95. This program carries out exact diagonalizations of truncated matrices and uses the following Hamiltonian:

$$
\hat{H}=\hat{H}_{\mathrm{r}}+\hat{H}_{\mathrm{e}}
$$

The first term, $\hat{H}_{\mathrm{r}}$, is the rigid-rotor Hamiltonian

$$
\hat{H}_{\mathrm{r}}=A J_{a}^{2}+B J_{b}^{2}+C J_{c}^{2}
$$

and $\hat{H}_{\mathrm{e}}$ is the Stark Hamiltonian

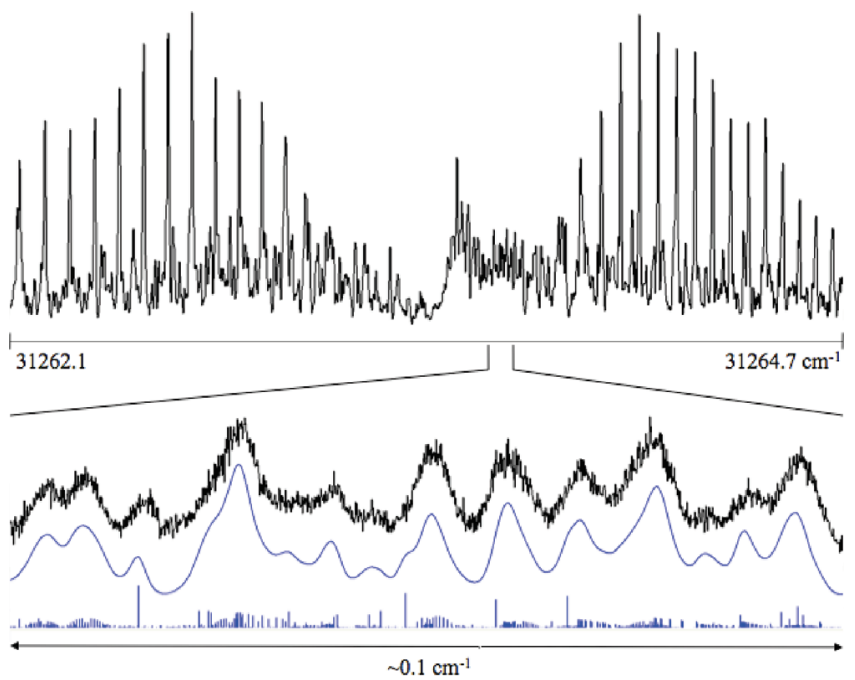

Figure 3. Origin band spectrum of $2 \mathrm{ABN}$, in presence of an electric field of $950 \mathrm{~V} / \mathrm{cm}$. The top panel shows the entire spectrum, and the bottom panel shows a comparison of a portion of the experimental spectrum (black trace) with two simulated spectra (blue trace), with and without a convoluted line shape function.

$$
\hat{H}_{\mathrm{e}}=-E_{Z} \sum_{g=a, b, c} \mu_{g} \phi_{Z_{g}}
$$

Here, $A, B$, and $C$ are the rotational constants, $J_{a}, J_{b}$, and $J_{c}$ are the projections of the angular momentum on the $a, b$, and $c$ inertial axes, $E_{Z}$ represents the electric field applied along the $Z$-axis, $\mu_{a}, \mu_{b}$, and $\mu_{c}$ are the dipole moment components along each inertial axis, and $\phi$ represents the direction cosines relating the lab and molecular frames. Other details have been described previously..$^{16}$ Application of an electric field causes transitions observed in the field-free spectrum to split and shift in frequency, as is apparent from eq 3 . The magnitudes of these shifts depend on both the field strength and dipole moment values.

By tracking the shifts of the spectral transitions in the presence of the external electric field, values of the dipole moment components of $2 \mathrm{ABN}$ were determined for both the ground and excited states. First, $\mu_{a}, \mu_{b}$, and $\mu_{c}$ were estimated using ab initio methods, and an initial simulation of the overall spectrum was created. The components were then varied until the simulation resembled the experimental spectrum. Next, lines were assigned, paying particular attention to those transitions that were most sensitive to the dipole moment components. The positions of $\mathrm{P}$ - and R-branch lines with high $K$ values, particularly those equal or nearly equal to $J$, are especially sensitive to $\mu_{a}$; the positions of the lines with $K_{a}=0$ are especially sensitive to $\mu_{c}$; the positions of Q-branch lines are sensitive to all three dipole moment components, especially $\mu_{b}$. The assigned transitions were then fit and used to create a new simulation, and the process was iterated until the simulation was in good agreement with the experiment. An example of the fit for $2 \mathrm{ABN}$ is shown in the lower portion of Figure 3. Typical OMC values of these fits are $\sim 6 \mathrm{MHz}$, substantially less than the line width.

Spectra of $3 \mathrm{ABN}$ taken with an applied electric field were fit in a manner similar to those of $2 \mathrm{ABN}$, see Figure 4. Relative to $2 \mathrm{ABN}$, the spectra of $3 \mathrm{ABN}$ were somewhat noisier, and the fits were characterized by slightly larger OMC values (12 $\mathrm{MHz}$ or less). Still, it proved possible to extract relatively precise values of $\mu$ in both electronic states of each molecule based on fits of their Stark spectra at five different field strengths.

Table 2 lists the derived values of $\mu$ that were obtained in this work. We note here that $\Delta \mu=\mu\left(\mathrm{S}_{1}\right)-\mu\left(\mathrm{S}_{0}\right)$ is relatively large for $3 \mathrm{ABN}$ and relatively small for $2 \mathrm{ABN}$. We also note
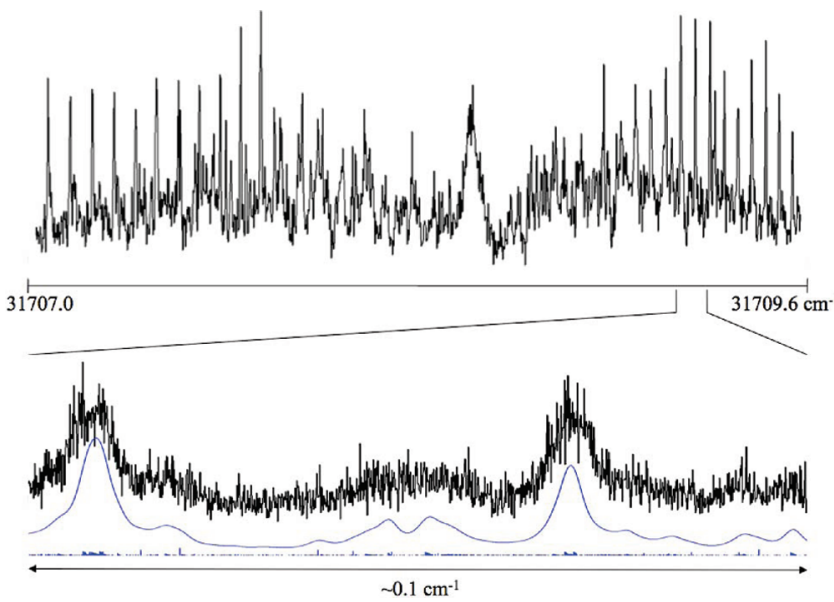

Figure 4. Origin band spectrum of $3 \mathrm{ABN}$, in presence of an electric field of $950 \mathrm{~V} / \mathrm{cm}$. The top panel shows the entire spectrum, and the bottom panel shows a comparison of a portion the experimental spectrum (black trace) with two simulated spectra (blue trace), with and without a convoluted line shape function. 
TABLE 2: Experimental Dipole Moment Values of 2ABN and $3 \mathrm{ABN}^{a}$

\begin{tabular}{|c|c|c|c|c|c|c|}
\hline & \multicolumn{3}{|c|}{$2 \mathrm{ABN}$} & \multicolumn{3}{|c|}{$3 \mathrm{ABN}$} \\
\hline & experiment & theory & $\begin{array}{c}\text { error } \\
(\%)\end{array}$ & experiment & theory & $\begin{array}{c}\text { error } \\
(\%)\end{array}$ \\
\hline \multicolumn{7}{|c|}{$\mathrm{S}_{0}$} \\
\hline$\mu_{a}(\mathrm{D})$ & $3.6(1)$ & 3.4 & 6 & $4.8(3)$ & 5.1 & 6 \\
\hline$\mu_{b}(\mathrm{D})$ & $1.9(2)$ & 1.7 & 10 & $1.2(4)$ & 0.6 & 50 \\
\hline$\mu_{c}(\mathrm{D})$ & 0.0 & 1.1 & & 0.0 & 1.2 & \\
\hline$\mu(\mathrm{D})$ & $4.1(2)$ & 4.0 & 2 & 4.9 (4) & 5.3 & 8 \\
\hline$\theta, a / b(\mathrm{deg})$ & 28 & 27 & & 14 & 7 & \\
\hline \multicolumn{7}{|c|}{$\mathrm{S}_{1}$} \\
\hline$\mu_{a}(\mathrm{D})$ & $3.4(2)$ & 3.9 & 15 & $6.8(3)$ & 8.1 & 19 \\
\hline$\mu_{b}(\mathrm{D})$ & $3.4(2)$ & 3.5 & 3 & 0.0 (4) & -0.4 & \\
\hline$\mu_{c}$ (D) & 0.0 & 0.0 & 0 & $0.0(1)$ & 0.0 & 0 \\
\hline$\mu(\mathrm{D})$ & $4.8(3)$ & 5.2 & 8 & $6.8(3)$ & 8.1 & 19 \\
\hline$\theta, a / b(\operatorname{deg})$ & 45 & 42 & & 0 & 3 & \\
\hline
\end{tabular}

${ }^{a}$ Theoretical values calculated using the MP2/6-31G** and CIS/ 6-31G** methods also have been included for comparison.

that the agreement between theory and experiment is poor, for both molecules in both electronic states. Differences in the values of $\mu_{c}$ are anticipated (see below). But the remaining calculated dipole moment components differ from the measured ones by as much as $50 \%$. Clearly, 6-31G** methods are inadequate to the task of predicting accurate values of the permanent electric dipole moments of isolated large molecules. (Abd El Rahim, et al. ${ }^{9}$ have reported improved values using larger basis sets.) Despite this fact, the discrepancies between the measured and calculated values are not large enough to explain the "anomalous" electric deflection behavior of the three aminobenzonitriles. ${ }^{9}$

Both theory and experiment agree that the $\mathrm{ABNs}$ have pyramidal $\mathrm{NH}_{2}$ groups, especially in their ground electronic states, necessitating nonzero values of $\mu_{c}$. But this localized structure is just one well of the double-minimum potential along this coordinate; the inverted structure has a value of $\mu_{c}$ which has the same magnitude but is opposite in sign. Ground-state values of $\mu_{c}$ less than $0.1 \mathrm{D}$ are required by our fits of the Stark
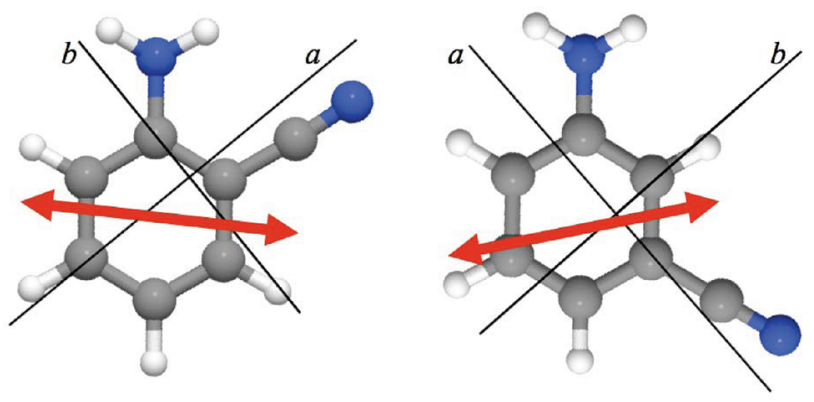

Figure 5. Structures of $2 \mathrm{ABN}$ and $3 \mathrm{ABN}$ with the $a$ and $b$ inertial axes shown for reference. The red arrows represent the predicted transition moment orientations.

behavior in ABNs. Since the calculated values are much larger than this (cf., Table 2), we conclude that motion along this coordinate is fast, compared to the frequency of rotational motion. A similar effect occurs in aniline, where the groundstate inversion barrier is of order $550 \mathrm{~cm}^{-1} \cdot{ }^{16,21,22}$

\section{Discussion}

Both $2 \mathrm{ABN}$ and $3 \mathrm{ABN}$ consist of a benzene ring to which amino $\left(-\mathrm{NH}_{2}\right)$ and nitrile $(-\mathrm{CN})$ substituents are attached. But the different relative placement of these substituents has several consequences for their spectra. Moving the amino group from the 2 - to the 3-position changes the orientation of the inertial axes and, consequently, the group's placement relative to the axis system of the molecule; see Figure 5. In $2 \mathrm{ABN}$, the amino group lies closest to the $b$-axis, but in $3 \mathrm{ABN}$, the amino group lies closest to the $a$-axis. The difference in the location of the axes is apparent on comparison of the ground-state rotational constants, given in Table 1. In 2ABN, the substituents are on opposite sides of the $a$-axis, whereas in $3 \mathrm{ABN}$ the substituents are on opposite sides of the $b$-axis. Therefore, $2 \mathrm{ABN}$ has the smaller value of $A$, whereas $3 \mathrm{ABN}$ has the smaller value of $B$. Overall, $2 \mathrm{ABN}$ has a more compact shape, resulting in a larger value of $C$.
LUMO+1

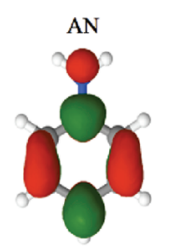

LUMO

номо
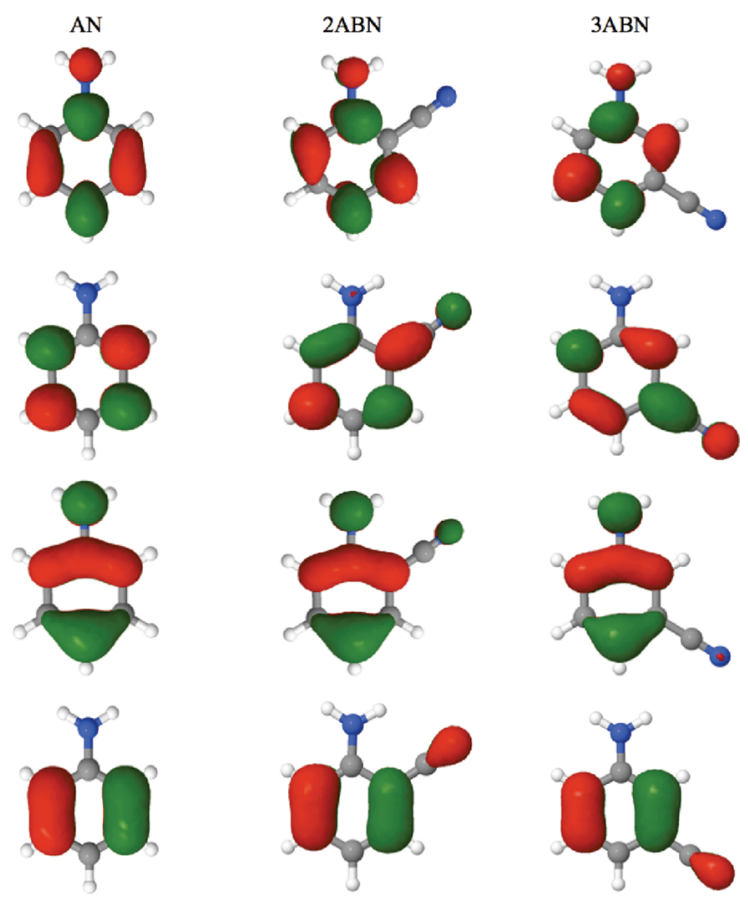
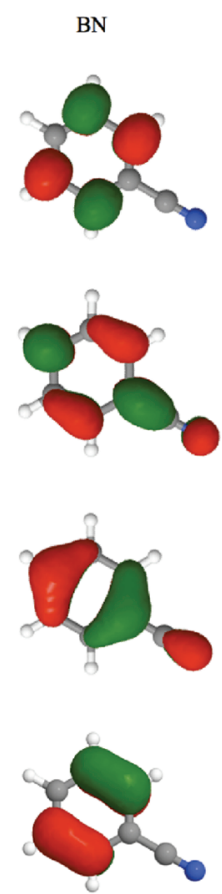

Figure 6. HOMO-1 $\left(\phi_{2}\right)$, HOMO $\left(\phi_{3}\right)$, LUMO $\left(\phi_{4}\right)$, and LUMO-1 $\left(\phi_{5}\right)$ molecular orbitals of AN, $2 \mathrm{ABN}, 3 \mathrm{ABN}$, and BN as predicted by CIS/6-31G** calculations. 
(a)

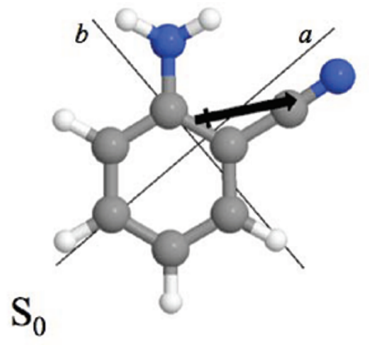

(b)

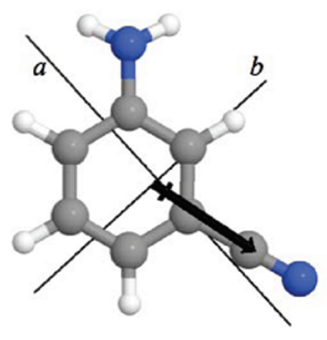

$\mathrm{S}_{0}$
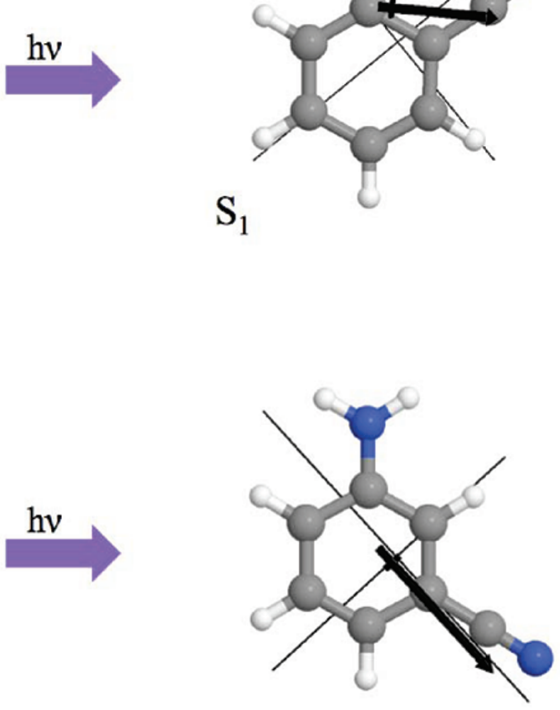

$\mathrm{S}_{1}$

Figure 7. Measured dipole moments are shown on the structures of $2 \mathrm{ABN}$ (a) and $3 \mathrm{ABN}(\mathrm{b})$ in both the ground and excited electronic states.

The appearances of the fully resolved electronic spectra of $2 \mathrm{ABN}$ and $3 \mathrm{ABN}$ also are quite different (Figure 3). The spectrum of $2 \mathrm{ABN}(45 / 55, a / b)$ has much more $a$-character than that of $3 \mathrm{ABN}(25 / 75, a / b)$; their transition moment (TM) orientations must be very different. Thus, it is possible that the electronic structures of the two molecules are quite different. Alternatively, it may be that these differences are more simply explained by differences in the orientations of their respective inertial axes.

CIS/6-31G** calculations ${ }^{20}$ were performed to address this issue. Figure 6 summarizes the results for aniline (AN), benzonitrile $(\mathrm{BN})$, and the two aminobenzonitriles, $2 \mathrm{ABN}$ and $3 \mathrm{ABN}$. Comparisons of the molecular orbitals (MOs) of the four molecules show that the highest occupied molecular orbital (HOMO) and HOMO-1 orbitals of $2 \mathrm{ABN}$ and $3 \mathrm{ABN}$ are very similar to those of AN. (There is some delocalization of charge onto the $-\mathrm{CN}$ substituent in the ABNs). Their lowest unoccupied molecular orbital (LUMO) and LUMO +1 orbitals are similar to those of both $\mathrm{AN}$ and $\mathrm{BN}$ but bear a closer resemblance to those of BN. Both AN and BN display $b$-type spectra. ${ }^{9,15}$ In CIS, the $S_{1}$ state of AN is represented by the linear combination $\Psi\left(S_{1}\right)=0.28\left(\phi_{2} \phi_{5}\right)+0.64\left(\phi_{3} \phi_{4}\right)$, whereas the $S_{1}$ state of $\mathrm{BN}$ is represented by the linear combination $\Psi\left(\mathrm{S}_{1}\right)=$ $0.55\left(\phi_{2} \phi_{4}\right)+0.41\left(\phi_{3} \phi_{5}\right)$. Similar calculations for the two ABNs yield the results $\Psi\left(S_{1}\right)=0.19\left(\phi_{2} \phi_{5}\right)+0.67\left(\phi_{3} \phi_{4}\right)$ for $2 \mathrm{ABN}$ and $\Psi\left(\mathrm{S}_{1}\right)=0.21\left(\phi_{2} \phi_{5}\right)+0.66\left(\phi_{3} \phi_{4}\right)$ for $3 \mathrm{ABN}$. Thus, the $-\mathrm{NH}_{2}$ group dominates in both cases; the $\mathrm{S}_{1}-\mathrm{S}_{0} \mathrm{TMs}$ take up orientations that are perpendicular to the amino group, and these orientations differ between the two molecules because of the difference in the orientations of their inertial axes; see Figure 5 . Thus, the electronic structures of both $2 \mathrm{ABN}$ and $3 \mathrm{ABN}$ are very similar, when viewed in this way.

Permanent dipole moments are more sensitive measures of the charge distributions in the two molecules and how these change when the photon is absorbed. Parts a and b of Figure 7 give visual images of these changes. Amino groups and cyano groups attached to an aromatic ring are electron-donating and electron-withdrawing, respectively. Thus, to a first approximation, the measured dipole moments of the two ABNs would be expected to be equal to the vector sums of the two substituent dipoles, $-\mathrm{NH}_{2}$ and $-\mathrm{CN}$, at least in the ground electronic state. This prediction was tested by forming the vector sums of the two dipoles using the measured dipole moments of $\mathrm{AN}^{16}$ and $\mathrm{BN}^{10}$ and the ab initio geometries of the two ABNs; the results are shown in Table 3. $2 \mathrm{ABN}$ has a predicted dipole moment of $4.0 \mathrm{D}$, compared to the measured value of $4.1 \mathrm{D}$; the resultant dipole vector makes a smaller angle with $a$ than with $b\left(\mu_{a} / \mu_{b}\right.$ $\sim 1.9)$. $3 \mathrm{ABN}$ has a predicted dipole moment of $5.1 \mathrm{D}$ compared

TABLE 3: Predicted and Measured Dipole Moments of 2ABN and 3ABN, Compared to the Previously Measured Values for AN, BN, and 4ABN, Based on the Vector Addition Model

\begin{tabular}{|c|c|c|c|c|c|c|c|c|}
\hline & \multirow[b]{2}{*}{$\mathrm{AN}^{a}$} & \multirow[b]{2}{*}{$\mathrm{BN}^{b}$} & \multicolumn{2}{|c|}{$2 \mathrm{ABN}$} & \multicolumn{2}{|c|}{$3 \mathrm{ABN}$} & \multicolumn{2}{|c|}{$4 \mathrm{ABN}^{b}$} \\
\hline & & & predicted & measured & predicted & measured & predicted & measured \\
\hline \multicolumn{9}{|c|}{$\mathrm{S}_{0}$} \\
\hline$\mu_{b}(\mathrm{D})$ & 0.00 & 0.00 & 1.6 & $1.9(2)$ & 0.7 & $1.2(4)$ & 0.00 & 0.00 \\
\hline$\mu(\mathrm{D})$ & 1.13 & 4.48 & 4.0 & $4.1(2)$ & 5.1 & $4.9(4)$ & 5.61 & $6.41(3)$ \\
\hline \multicolumn{9}{|c|}{$\mathrm{S}_{1}$} \\
\hline$\mu(\mathrm{D})$ & 2.80 & 4.57 & 3.7 & $4.8(3)$ & 6.5 & $6.8(3)$ & 7.37 & $7.20(3)$ \\
\hline
\end{tabular}

${ }^{a} \operatorname{Ref} 16 .{ }^{b} \operatorname{Ref} 10$. 


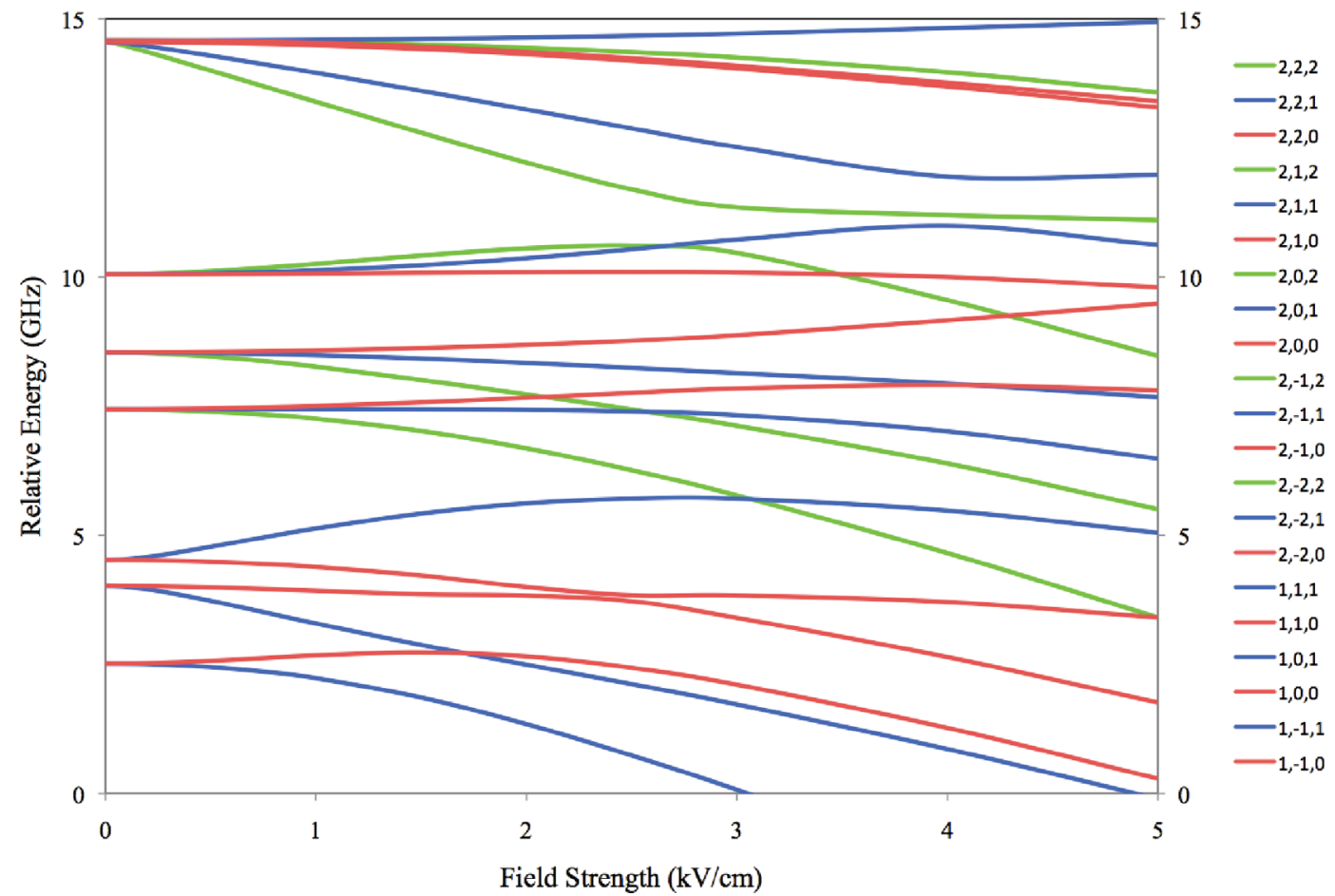

Figure 8. Stark-splitting energy level diagram for the ground-state $J=1$ and 2 rotational levels of $2 \mathrm{ABN}$ as a function of electric field. The simulation was run with the experimentally determined rotational constants and dipole moments. The labels on the right designate the $J, \tau$, and $M$ values of the represented levels.

to the measured value of $4.9 \mathrm{D}$; the resultant dipole vector makes an even smaller angle with $a$ than with $b\left(\mu_{a} / \mu_{b} \sim 4\right)$. Thus, to a reasonable approximation, the dipole moments of $2 \mathrm{ABN}$ and $3 \mathrm{ABN}$ are the vector sums of the two component dipoles, in the ground electronic state. A similar conclusion has been reached for $4 \mathrm{ABN} .^{10}$

Excitation of both molecules to their $S_{1}$ states leads to large increases in both the magnitudes and orientations of their dipole moment vectors. In $2 \mathrm{ABN}$, the increase in the magnitude of $\mu$ (4.8 $\mathrm{D}$ in the $\mathrm{S}_{1}$ state) is caused by large increase in $\mu_{b}$ and a small decrease in $\mu_{a}$, effects that are nicely captured by the vector addition model. The $-\mathrm{NH}_{2}$ group is a stronger donor, and the $-\mathrm{CN}$ group is a stronger acceptor, in the $\mathrm{S}_{1}$ state. In $3 \mathrm{ABN}$, owing to the aforementioned change in the inertial axes, the larger value of $\mu$ (6.8 D in the $S_{1}$ state) is caused by a large increase in $\mu_{a}$ and a small decrease in $\mu_{b}$, again reflecting the stronger electron donor/acceptor character of the $-\mathrm{NH}_{2} /-\mathrm{CN}$ groups. More striking is the change in orientation of the dipole moment vectors; these change by $\pm 15^{\circ}$ when the photon is absorbed. Large solvatochromic shifts of the fluorescence spectra of both molecules would be expected in the condensed phase. ${ }^{23}$

Deviations from the "additivity rule" are most pronounced for the $S_{0}$ state of $3 \mathrm{ABN}$, where the predicted value is high, and for the $S_{1}$ state of $2 A B N$, where the predicted value is low. These effects may be traced to the MOs of the two molecules shown in Figure 6. As noted, although similar to the orbitals of $\mathrm{AN}$ and $\mathrm{BN}$, the $\mathrm{HOMO}$ and $\mathrm{HOMO}-1$ orbitals of the ABNs more closely resemble those of AN, whereas the LUMO and $\mathrm{LUMO}+1$ orbitals bear a stronger resemblance to BN. Hence, it is reasonable that the dipole moment components are not strictly additive across the entire series of molecules.

Previous attempts to measure the permanent electric dipole moments of the three structural isomers of $\mathrm{ABN}$ by molecular beam deflection methods have led to ambiguous results. ${ }^{9}$ Increasing deviations of the measured deflections compared to Stark-effect calculations were observed in the series $4 \mathrm{ABN}$,
$3 \mathrm{ABN}$, and $2 \mathrm{ABN}$. These deviations were attributed to the increasing asymmetry of the molecules across the series. To support this view, it was shown by first-principles calculations that the Stark-split energy levels of asymmetric tops begin to repel each other at high fields. These level repulsions lead to a mixing of rotational energy levels and a reduced energy shifting of the eigenvalues as the electric field is increased. That large molecules (whether asymmetric or not) excited to single rovibrational eigenstates are not deflected by an inhomogeneous electric field in a high density-of-states region was earlier established by the elegant infrared experiments of Fraser and Pate, ${ }^{11}$ so the results of Abd El Rahim et al. ${ }^{9}$ are not surprising.

Although the ABNs are certainly asymmetric (the $\kappa$ values of $2 \mathrm{ABN}, 3 \mathrm{ABN}$, and $4 \mathrm{ABN}$ in their electronic ground states are $-0.50,-0.74$, and -0.94 , respectively), their Stark-split energy levels are not dominated by avoided crossings at low field. This is shown explicitly for $2 \mathrm{ABN}$ in Figure 8. In fields up to $5 \mathrm{kV} / \mathrm{cm}$, only three avoided crossings appear in the plots of state energy versus field at low $J$. Even fewer appear at higher $J$, at low field strengths. Thus, the energy levels of both symmetric and asymmetric rotors are regular, not irregular, at low field strengths. Higher field strengths do lead to extensive level mixing, forming the basis for a number of creative applications, including studies of the dynamics of molecules in aligned rotational states ("pendular" states") ${ }^{24}$ and the spatial separations of the structural isomers of neutral molecules according to their mass-to-dipole-moment ratios. ${ }^{25}$ But, as the present application shows, lower field strengths are also useful. Selective excitation of the Stark-split spectra of large molecules leads to an unambiguous determination of the magnitude and orientation of their permanent dipole moments in different electronic states. Further experiments will exploit this fact in studies of the charge distributions of biologically relevant molecules in the gas phase and how they are affected by the absorption of light. 
Acknowledgment. This work was supported by NSF (CHE0615755). We thank A. J. Fleisher and R. A. Christie for assistance and useful discussions, D. F. Plusquellic for helping with Figure 8, and the Center for Molecular and Material Simulations at the University of Pittsburgh for computing time. We also are especially grateful to Robert Zalesny from the Wroclaw University of Technology for advice on the dipole moment calculations.

\section{References and Notes}

(1) Berg, J. M.; Tymoczko, J. L.; Stryer, L. Biochemistry, 6th ed.; W. H. Freeman and Co.: San Francisco, CA, 2006

(2) Tartaglia, G. G.; Cavalli, A.; Pellarin, R.; Caflisch, A. Protein Sci. 2004, 13, 1939.

(3) (a) Brown, R. D.; Godfrey, P. D.; Stovey, J. W. V.; Bassez, M.-P. J. Chem. Soc., Chem. Commun. 1978, 547. (b) Vogelsanger, B.; Godfrey, P. D.; Brown, R. D. J. Am. Chem. Soc. 1991, 113, 7864. (c) Godfrey, P. D.; Firth, S.; Hatherley, L. D.; Brown, R. D.; Pierlot, A. P. J. Am. Chem. Soc. $1993,115,9867$.

(4) See, for example, Sanz, M. E.; Lesari, A.; Peña, M. I.; Vaquero, V.; Cortijo, V.; Lopez, J. C.; Alonso, J. L. J. Am. Chem. Soc. 2006, 128, 3812.

(5) See, for example, Gloaguen, E.; Pagliarulo, F.; Breuner, V.; Chin, W.; Piuzzi, F.; Tardivel, B.; Mons, M. Phys. Chem. Chem. Phys. 2007, 9, 4491.

(6) Compagnon, I.; Hagemeister, F. C.; Antoine, R.; Rayane, D.; Broyer, M.; Dugourd, P.; Hudgins, R. R.; Jarrold, M. F. J. Am. Chem. Soc. 2001, 123, 8440

(7) Antoine, R.; Compagnon, I.; Rayane, D.; Broyer, M.; Dugourd, P.; Breaux, G.; Hagemeister, F. C.; Pippen, D.; Hudgins, R. R.; Jarrold, M. F. J. Am. Chem. Soc. 2002, 124, 6737.

(8) Dugourd, P.; Antoine, R.; Breaux, G.; Broyer, M.; Jarrold, M. F. J. Am. Chem. Soc. 2005, 127, 4675.

(9) Abd El Rahim, M.; Antoine, R.; Broyer, M.; Rayane, D.; Dugourd, P. J. Phys. Chem. A 2005, 109, 8507

(10) Borst, D. R.; Korter, T. M.; Pratt, D. W. Chem. Phys. Lett. 2001, 350,485

(11) (a) Fraser, G. T.; Pate, B. H. J. Chem. Phys. 1994, 100, 6210 and references therein, especially,(b) Fraser, R. G. J. Molecular Beams; Methuen and Co.: London, 1937. See also (c) Lombardi, J. R. J. Chem. Phys. 1968, 48,348 .
(12) Majewski, W. A.; Pfanstiel, J. F.; Plusquellic, D. F.; Pratt, D. W. In Laser Techniques in Chemistry; Myers, A. B., Rizzo, T. R., Eds.; J. Wiley \& Sons: New York, 1995.

(13) Miller, D. M. Ph.D. Thesis, University of Pittsburgh, 2009.

(14) Plusquellic, D. F. Ph.D. Thesis, University of Pittsburgh, 1992.

(15) Plusquellic, D. F.; Suenram, R. D.; Maté, B.; Jensen, J. O.; Samuels, A. C. J. Chem. Phys. 2001, 115, 3057.

(16) Korter, T. M.; Borst, D. R.; Butler, C. J.; Pratt, D. W. J. Am. Chem. Soc. 2001, 123, 96.

(17) Kolek, P.; Pirowska, K.; Najbar, J. Phys. Chem. Chem. Phys. 2001, 3,4874 .

(18) Howell, R.; Joslin, E. M.; Taylor, A. G.; Phillips, D. J. Chem. Soc., Faraday Trans. 1992, 88, 1605.

(19) Herzberg, G. Electronic Spectra of Polyatomic Molecules; D. van Nostrand: Princeton, NJ, 1966.

(20) Frisch, M. J.; Trucks, G. W.; Schlegel, H. B.; Scuseria, G. E.; Robb, M. A.; Cheeseman, J. R.; Montgomery, J. A., Jr.; Vreven, T.; Kudin, K. N.; Burant, J. C.; Millam, J. M.; Iyengar, S. S.; Tomasi, J. J.; Barone, V.; Mennucci, B.; Cossi, M.; Scalmani, G.; Rega, N.; Petersson, G. A.; Nakatsuji, H.; Hada, M.; Ehara, M.; Toyota, K.; Fukuda, R.; Hasegawa, J.; Ishida, M.; Nakajima, T.; Honda, Y.; Kitao, O.; Nakai, H.; Klene, M.; Li, X.; Knox, J. E.; Hratchian, H. P.; Cross, J. B.; Adamo, C.; Jaramillo, J.; Gomperts, R.; Stratmann, R. E.; Yazyev, O.; Austin, A. J.; Cammi, R.; Pomelli, C.; Ochterski, J. W.; Ayala, P. Y.; Morokuma, K.; Voth, A.; Salvador, P.; Dannenberg, J. J.; Zakrzewski, V. G.; Dapprich, S.; Daniels, A. D.; Strain, M. C.; Farkas, O.; Malick, D. K.; Rabuck, A. D.; Raghavachari, K.; Foresman, J. B.; Ortiz, J. V.; Cui, Q.; Baboul, A. G.; Clifford, S.; Cioslowski, J.; Stefanov, B. B.; Liu, G.; Liashenko, A.; Piskorz, P.; Komaromi, I.; Martin, R. L.; Fox, D. J.; Keith, T.; Al-Laham, M. A.; Peng, C. Y.; Nanayakkara, A.; Challacombe, M.; Gill, P. M. W.; Johnson, B.; Chen, W.; Wong, M. W.; Gonzalez, C.; Pople, J. A. Gaussian 03, revision 6.0; Gaussian, Inc.: Wallingford, CT, 2004

(21) A nonzero value of $\mu_{c}$ would also connect the even and odd symmetry levels of an inverting molecule, producing additional Stark shifts in the spectra (ref 22). However, given the anticipated large separation of these levels, and the experimental resolution, we consider this unlikely.

(22) Gordy, W.; Cook, R. L. Microwave Molecular Spectra; J. Wiley and Sons: New York, 1984.

(23) Jiang, S.; Levy, D. H. J. Phys. Chem. A 2003, 107, 6785.

(24) Friedrich, B.; Herschbach, D. R. Nature 1991, 353, 412.

(25) Filsinger, F.; Erlekam, U.; Von Helden, G.; Kuepper, J.; Meijer, G. Phys. Rev. Lett. 2008, 100, 133003.

JP9017585 